\title{
Comparison of Oblique Lateral Interbody Fusion and Transforaminal Lumbar Interbody Fusion for Degenerative Lumbar Disease: A Meta-Analysis
}

CHEN Tai-bang ( $\nabla$ chentaibang123@sina.com )

Kunming General Hospital of Chengdu Military Region https://orcid.org/0000-0003-2071-5161

HE Xiao-qing

Institute of trauma and orthopedics, the No.920 hospital of joint logistics support force of PLA

Liang Jing-long

institute of trauma and orthopedics, the No.920 hospital of joint logistics support force of PLA

Research article

Keywords: Oblique Lateral Interbody Fusion, Transforaminal Lumbar Interbody Fusion, Meta-Analysis

Posted Date: September 4th, 2020

DOI: https://doi.org/10.21203/rs.3.rs-70799/v1

License: (c) (1) This work is licensed under a Creative Commons Attribution 4.0 International License.

Read Full License 


\section{Abstract}

Aim: Both oblique lateral interbody fusion (OLIF) and transforaminal lumbar interbody fusion (TLIF) are frequently used to treat degenerative lumbar disease. The purpose of this meta-analysis is to compare the radiologic and clinical outcomes between these two methods.

Methods: Electronic databases, including PubMed, Web of Science and MEDLINE, were searched to identify relevant studies that compared OLIF and TLIF up to May 2020. The radiographic outcomes comprised of the disc height (DH), lumbar lordotic angle (LLA), disc angle (DA), fusion rate (FR), and foraminal height $(\mathrm{FH})$. The secondary outcomes were length of hospital stay, operation time, estimated blood loss, visual analog scale (VAS), and Oswestry Disability Index (ODI). Data pooling and a metaanalysis with the random effects model were performed to evaluate the major results and conclusions.

Results: A total of nine studies that involved 593 patients (271 patients in the OLIF group and 322 in the TLIF group) were included in the meta-analysis. Similar changes, in terms of disc height and fusion rate of $>80 \%$, were observed between the two groups. In comparing OLIF to TLIF, the OLIF group had less estimated blood loss, and a shorter operative time and hospital stay, with statistical difference. However, there was no significant difference in VAS and ODI between OLIF and TLIF.

The meta-analysis suggested that TLIF is associated with better postoperative LLA, FH and DA, when compared to OLIF. However, these were not statistically significant $(P>005)$.

Conclusion: These results demonstrate that both OLIF and TLIF are similar in terms of the restoration of disc height and intervertebral fusion rate in the treatment of degenerative lumbar diseases. OLIF was superior to TLIF in terms of operation time, hospital stay and estimated blood loss. However, there was no advantage in restoring the sagittal balance and correcting the lordosis.

\section{Introduction}

Lumbar fusion is an effective surgical intervention for degenerative lumbar disease, which is widely used in clinical practice. The procedure is usually performed using five main techniques: posterior lumbar interbody fusion (PLIF), transforaminal lumbar interbody fusion (TLIF or MI-TLIF), oblique lumbar interbody fusion/anterior to psoas (OLIF/ATP), anterior lumbar interbody fusion (ALIF), and lateral lumbar interbody fusion (LLIF) [1].

TLIF was described via the transforaminal route to achieve the insertion of an interbody cage packed with bone graft [2]. At present, more and more spinal surgeons prefer this approach, because they can reduce the dural retraction and enable direct neural decompression. A study suggested that TLIF is superior to PLIF in terms of operation time, less blood loss, and lower incidence of nerve root injury [3]. Although the method had satisfactory outcomes, iatrogenic complications, such as nerve injury and the stripping of paravertebral muscles that result in a long-lasting sequelae, cannot be avoided. In order to minimize trauma, a minimally invasive retroperitoneal lumbar approach was introduced by Mayer in 1997 [4]. 
However, the name of OLIF was first described in 2012 [5]. This retroperitoneal approach involved access to the disc space via a corridor between the peritoneum and psoas muscle, which allows for the efficient clearance of the intervertebral disc space and the insertion of large cages. OLIF does not require posterior surgery, laminectomy, facetectomy, and stripping of the spinal paraspinal musculature. The OLIF approach was gradually accepted by clinicians.

To date, both OLIF and TLIF have been advocated, but there is still controversy over the best surgical technique due to the inconsistent published results. Therefore, the investigators systematically search and analyze the existing literature to evaluate the safety and effectiveness between these two methods in the treatment of lumbar degenerative diseases.

\section{Materials And Methods}

\section{Literature Search}

PubMed, Web of Science and MEDLINE were used to conduct a comprehensive search of published literature. The search terms included the following: "TLIF" or "transforaminal lumbar interbody fusion", "OLIF" or "transforaminal lumbar interbody fusion" or "degenerative lumbar disease". The publication language was not restricted. The "related articles" function and manual search of the reference list were also used to expand the search scope.

\section{Inclusion and Exclusion criteria}

The following inclusion criteria were used: a randomized controlled trial (RCT) or retrospective comparative study design (cohort or case-controlled studies) that compared OLIF and TLIF in the treatment of degeneration lumbar disease. Incorporating the literature can provide the clinical and radiographic results of these two procedures, and exclude editorials, letters, review articles, case reports, and animal studies.

\section{Data Extraction}

Two authors independently extracted the data from eligible studies, and any disputes were resolved either by discussion or by involving a third author, when necessary. The information extraction from each study was, as follows: (1) author and publication year; (2) sample size; (3) fusion rate (FR); (4) operation time; (5) estimated blood lose; (6) hospital stay; (8) foraminal height(FH); (9) disc angle (DA); (10) lumber lordosis angle(LLA); (11) Oswestry Disability Index(ODI); (12) Visual Analogue Scale(VAS).

\section{Statistical Analysis}

For the pooled effects, the weighted mean difference (WMD) or standard mean difference (SMD) was calculated for continuous variables, according to the consistency of the measurement units, and odds ratio (OR) was calculated for dichotomous variables. For all these comparisons, a $P$-value of $<0.05$ was considered statistically significant. The investigators evaluated the heterogeneity between articles using 
the $\mathrm{I}^{2}$ statistic. The heterogeneity between articles was assessed to be greater with a higher $\mathrm{I}^{2}$ statistic. When $\mathrm{I}^{2} \geq 50 \%$, the assumption of homogeneity was rejected, and a random-effects model was adopted. Otherwise, a fixed-effects model was used.

\section{Results}

\section{Database results}

The flow diagram of the search strategy is summarized in Fig.\&\#x00A0;1. Initially, 551 articles on the comparative study of OLF and TLIF were searched in the electronic database. The remaining 25 studies were read in full, and 16 studies were excluded due to unavailable selection criteria. Finally, a total of nine articles that involved 593 patients (271 patients in the OLIF group and 322 patients in the TLIF group) met the inclusion criteria, and were included in the present meta-analysis [6\&\#x2013;14]. Among the included articles, six articles were in the English language, while the other articles were in the Chinese language.

\section{Demographic Results}

The characteristics of the included studies are presented in Table\&\#x00A0;1. The bias risk and quality of the included studies were assessed using the Newcastle-Ottawa Scale (NOS). The NOS contains eight scoring items categorized into three fields: Selection, Comparability, and Outcome. The full score was 9 , and studies that achieved a score of 6 or more were considered as high quality. Among these studies, five studies scored 8 points, while the remaining studies scored 7 points. These studies are of high quality, as presented in Table 2. 
Table 1. Characteristics of the included studies

\begin{tabular}{|lclllll|}
\hline Study & Year & Study type & $\begin{array}{l}\text { Gender } \\
\text { distribution } \\
\text { (male/female) }\end{array}$ & $\begin{array}{l}\text { Sample } \\
\text { size } \\
\text { (OLIF/TLIF) }\end{array}$ & $\begin{array}{l}\text { Newcastle- } \\
\text { Ottawa } \\
\text { score }\end{array}$ & $\begin{array}{l}\text { Follow-up } \\
\text { time } \\
\text { (month) }\end{array}$ \\
$\begin{array}{l}\text { Seung } \\
\text { Won Park }\end{array}$ & 2020 & $\begin{array}{l}\text { Retrospective } \\
\text { cohort }\end{array}$ & $3 / 20$ & $13 / 10$ & 8 & $36-55$ \\
$\begin{array}{l}\text { Yoshinao } \\
\text { Koike }\end{array}$ & 2020 & $\begin{array}{l}\text { Retrospective } \\
\text { study }\end{array}$ & $38 / 48$ & $38 / 48$ & 8 & $12-24$ \\
\hline $\begin{array}{l}\text { Yi-Lei } \\
\text { Chen }\end{array}$ & 2018 & $\begin{array}{l}\text { Retrospective } \\
\text { study }\end{array}$ & $31 / 42$ & $34 / 39$ & 8 & $5-18$ \\
$\begin{array}{l}\text { Hah Yong } \\
\text { Mun }\end{array}$ & 2019 & $\begin{array}{l}\text { Retrospective } \\
\text { study }\end{array}$ & $44 / 104$ & $74 / 74$ & 8 & $5-23$ \\
$\begin{array}{l}\text { Guang- } \\
\text { Xun Lin }\end{array}$ & 2018 & $\begin{array}{l}\text { Retrospective } \\
\text { study }\end{array}$ & $16 / 34$ & $25 / 25$ & 7 & $20-55$ \\
\hline $\begin{array}{l}\text { Shi-Qiang } \\
\text { Qin }\end{array}$ & 2018 & RCT & $30 / 38$ & $34 / 34$ & 7 & $10-18$ \\
\hline $\begin{array}{l}\text { Sun-Ren } \\
\text { Sheng }\end{array}$ & 2020 & $\begin{array}{l}\text { Retrospective } \\
\text { study }\end{array}$ & $33 / 60$ & $38 / 55$ & 8 & 12 \\
\hline $\begin{array}{l}\text { Qiang } \\
\text { Zhang }\end{array}$ & 2019 & RCT & $13 / 17$ & $15 / 15$ & 7 & 12 \\
\hline $\begin{array}{l}\text { Wei- } \\
\text { Zhuang } \\
\text { Guo }\end{array}$ & 2019 & RCT & $41 / 45$ & $45 / 45$ & 7 & 12 \\
\hline
\end{tabular}

Table 2. Newcastle-Ottawa scale 


\begin{tabular}{|c|c|c|c|c|c|c|c|c|}
\hline \multirow[t]{2}{*}{ Studies } & \multicolumn{4}{|c|}{ Selection } & $\begin{array}{l}\text { Comparabilit } \\
y\end{array}$ & \multicolumn{3}{|c|}{ Exposure } \\
\hline & $\begin{array}{l}\text { Case } \\
\text { definitio } \\
\text { n }\end{array}$ & $\begin{array}{l}\text { Representativenes } \\
\mathrm{s}\end{array}$ & $\begin{array}{l}\text { Control } \\
\text { selectio } \\
\text { n }\end{array}$ & $\begin{array}{l}\text { Control } \\
\text { definitio } \\
n\end{array}$ & & $\begin{array}{l}\text { Ascertainmen } \\
\text { t }\end{array}$ & $\begin{array}{l}\text { Same method } \\
\text { of } \\
\text { ascertainmen } \\
t \text { for cases } \\
\text { and controls }\end{array}$ & $\begin{array}{l}\text { Nonreapons } \\
\text { e rate. }\end{array}$ \\
\hline Chen & 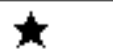 & $\star t$ & t & th & th & & 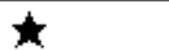 & 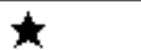 \\
\hline Lin & $t$ & 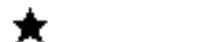 & t & t & $t$ & & t & \\
\hline Park & 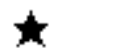 & $\star t$ & th & t & $t k$ & & t & th \\
\hline Qin & $\star$ & $\star$ & th & th & 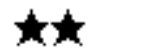 & & t & \\
\hline Sheng & th & t & th & th & $\not t$ & & $\star t$ & \\
\hline Guo & $t$ & 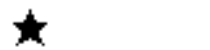 & th & th & $\Delta+$ & & $\star t$ & t \\
\hline Koike & $\star$ & $\star$ & t & t & $\Delta+$ & & $\star$ & 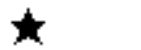 \\
\hline Zhan & th & th & th & th & 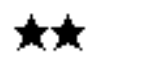 & & t & \\
\hline $\mathrm{g}$ & & & & & & & & \\
\hline Mum & t & t & th & th & $\Delta k$ & & 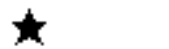 & t \\
\hline
\end{tabular}

\section{Operation Time}

The data of eight studies, which included 513 patients (242 patients in the OLIF group and 271 patients in the TLIF group) was analyzed. The pooled estimate revealed that there was a statistical difference between both groups, in terms of operative time, in favor of the shorter operative times for the OLIF group. $[S M D=-1.29,95 \% \mathrm{Cl}(-2.12,-0.47), \mathrm{P}<0.05 ;$ Fig. 2]. This meta-analysis involved a random-effects model due to the high heterogeneity among the studies $\left(I^{2}=94 \%\right)$.

\section{Estimated Blood Lose}

The estimated blood loss obtained in eight studies that involved 513 patients (242 patients in the OLIF group and 271 patients in the TLIF group) was analyzed. The result of the meta-analysis indicated that the TLIF group had a significantly higher estimated blood loss, when compared to the OLIF group, with significant heterogeneity $[\mathrm{P}<0.00, \mathrm{MD}=-141.09$ (-183.44, -98.74$) ; I^{2}=97 \%$; Fig. 3].

\section{Hospital Stay}

Three studies, which included 211 patients (97 patients in the OLIF group and 114 patients in the TLIF group), reported the data on hospital stays. The meta-analysis results revealed that TLIF required a significantly longer hospital stay, when compared to OLIF $[P<0.05, S M D=-1.72(-3.29,-0.15)]$. This metaanalysis involved a random-effects model due to the high heterogeneity among studies $\left(I^{2}=70 \%\right)$. The details for each study are shown in Figure 4. 
Among the nine articles, six studies compared the ODI. The forest plot for the ODI between OLIF and TLIF is presented in Figure 4. The meta-analysis indicated that OLIF was superior to TLIF, but the difference was not statistically significant $[P=0.40, M D=-0.52(-1.73,-0.69)$; Fig. 5], with a detected significant heterogeneity $\left(I^{2}=79 \% ; P=0.0002\right)$.

\section{VAS}

Six of nine included studies involved the VAS. The forest plot for the VAS between OLIF and TLIF is shown in Figure 5. The combined results suggest that the score of VAS in the OLIF is lower than that in the TLIF, but there was no significant difference $[P=0.67, M D=-0.01(-0.34,-0.22)]$. Evidence of statistically significant heterogeneity was observed $\left[I^{2}=83 \%, P<0.0001 ;\right.$ Fig. 6].

\section{Radiographic Results}

The investigators also compared the intervertebral disc height, foramen height, fusion rate, lumbar lordosis Angle and intervertebral disc angle between the two groups after the operation. A total of five component studies, which included 407 patients (190 patients in the OLIF group and 217 patients in the TLIF group), provided the value of the disc height. Disc height in the OLIF was similar to that in the TLIF $\left[\mathrm{MD}=1.17,95 \% \mathrm{Cl}(0.29-2.05), \mathrm{P}=0.009 ; \mathrm{I}^{2}=91 \%\right.$; Fig. 7]. Four studies, which involved 196 patients (97 patients in the OLIF and 99 patients in the TLIF), investigated the data for the lumbar lordosis angle. Compared with OLIF group, the TLIF group was associated with a much more improved lumbar lordosis angle after the operation, with no statistical difference and significant heterogeneity $[\mathrm{MD}=2.18,95 \% \mathrm{Cl}$ (-1.49 5.85), $P=0.24 ; I^{2}=87 \%$; Fig. 8]. Four studies, which included 337 patients, compared the effects of OLIF and TLIF on the disc angle. The TLIF had a greater recovery of the disc angle than that of the OLIF, but there was no significant difference with large heterogeneity between groups [MD $=5.33,95 \% \mathrm{Cl}$ (-0.92 11.58), $\mathrm{P}=0.09 ; \mathrm{I}^{2}=98 \%$; Fig. 9]. Three studies, which included 288 patients, reported the data on the fusion rate. The meta-analysis results revealed that the postoperative fusion rates were similar between the two groups, but there was no statistical difference [MD=1.55, 95\% $\mathrm{Cl}(-0.47-5.1), \mathrm{P}=0.09$; Fig. 10]. This meta-analysis involved a random-effects model due to the high heterogeneity among studies $\left(I^{2}=67 \%\right)$. A total of 321 patients from four studies compared the recovery of the foramina height between OLIF and TLIF. The combined data revealed that the recovery of the foramina height in the OLIF was higher than in the TLIF, but there was no statistical difference [MD $=-0.10,95 \% \mathrm{Cl}(-0.19 \sim-0.00), \mathrm{P}=0.05 ; \mathrm{I}^{2}$ $=0 \%$; Fig. 11].

\section{Discussion}

TLIF has become a prevalent and well-established surgical procedure for the treatment of lumbar degenerative disease since its description was reported by Harms et al. [15]. Although the technique had high rates of success, iatrogenic complications, such as nerve injury and the stripping of the paravertebral muscles that resulted in a long-lasting sequelae, cannot be avoided. Dural tear is the most common intraoperative complication. Other intraoperative complications included neurological injury and 
endplate injury. The OLIF approach has been used as an anatomical pathway through the retroperitoneal space between the psoas muscle and large abdominal vessels. The advantage of OLIF is to avoid the nerve root damage and dural tear caused by posterior surgery, without destroying the articular process joints, interspinous ligament, and supraspinous ligament [16]. The impact on spinal stability and posterior structure was minimized. The most common complication of OLIF was peritoneal laceration, sympathetic chain and vascular and injuries [17]. Although there are related studies that compared OLIF with TLIF, the evidence that OLIF is superior to TLIF remains insufficient. In order to address this uncertainty, the investigators conducted a meta-analysis to compare the radiographic and clinical outcomes of OLIF and TLIF for degenerative lumbar disease, providing effective evidence for clinicians.

As of the present literature search, a previous meta-analysis conducted by Lin compared OLIF and MITLIF in the treatment of degenerative lumbar disease in October 2018 [18]. The analysis included 47 studies that involved 3,587 patients, and a systematic review of related studies was performed. The results suggested that the radiographic and functional outcomes and length of hospital stay are similar between these two groups. The OLIF group exhibited the advantages in operative blood loss and operative time. However, the study conducted by the authors only performed within-group comparisons for clinical and radiographic outcomes before and after the operation, without between-group. Therefore, the meta-analysis cannot draw convincing conclusions. The present study was a meta-analysis of eligible studies with the objective of identifying the safety and effectiveness of OLIF and TLIF after the degenerative lumbar disease surgery.

It has been generally assumed that neural function improvement is relative to the clinical outcomes after the degeneration spinal disease surgery. The present study was identified with this view. The VAS and ODI were the important parameter to appraise the life quality. The pooled estimates suggested that satisfactory clinical results could be achieved in the two groups. Surprisingly, although there was no significant difference between both groups, in terms of postoperative VAS and ODI, these results were better in the OLIF group, when compared with the TLIF group. OLIF is superior to TLIF in terms of operation time, hospital stay, and estimated blood loss. A possible explanation for this might be that TLIF requires posterior surgery, laminectomy, facetectomy, or stripping of the spinal or paraspinal musculature, resulting in a long-lasting sequelae. Furthermore, OLIF avoided the destruction of the articular process joints, interspinous ligament and supraspinous ligament, reducing the probability of chronic low back pain.

The sagittal balance correction in the lumbar spine surgery is relevant to greater postoperative outcomes. Various interbody fusion procedures were conducted to correct the sagittal balance, with no scientific consensus, in which the technique offers better correction [19]. The combined results show that the recovery of the disc height was similar between the two groups. However, there were no significant statistical differences in the lumbar lordosis angle, intervertebral disc angle, foramen height, and fusion rate between the two groups. From the meta-analysis of the clinical and radiographic outcomes, it was suggested that the intervertebral foramen height, lumbar lordosis angle and intervertebral disc angle 
should not affect the short-term clinical outcomes of patients with OLIF and TLIF. However, the restoration of disc height might affect the patient\&\#x2019;s postoperative satisfaction.

The TLIF approach can reduce the dural retraction, and enable direct neural decompression, achieving the intended goals of surgery (pain and functional improvement, decompression of the spinal canal and the foramen, and restoration of the sagittal and coronal alignment). OLIF obtains the comparable outcomes of TLIF through indirect decompression, in terms of accomplishing the intended surgical goal. It has been generally considered that the limitation of OLIF with indirect decompression may be spinal pathologies combined with calcified disc coupled with bony spur formation, severe lateral recess stenosis, facet arthrosis, or synovial cysts, which cannot be relieved by indirect decompression. The advantage of OLIF is that it can indirectly achieve decompression and preserve the posterior column structure, correct the coronal and sagittal imbalance, reduce the paraspinal muscle trauma, minimize blood lose, shorten the hospital stay, and result in faster patient recovery with fewer complications.

\section{Conclusion}

These present results demonstrate that both OLIF and TLIF are similar in terms of the restoration of disc height and intervertebral fusion rate in the treatment of degenerative lumbar diseases. OLIF is superior to TLIF with regards to operation time, hospital stay and estimated blood loss, but there was no advantage in restoring the sagittal balance and correcting the lordosis. The meta-analysis suggests that TLIF is associated with better postoperative LLA, FH and DA, when compared to OLIF. However, these were not statistically significant. This suggests that the intervertebral foramen height, lumbar lordosis angle, and intervertebral disc angle should not affect the short-term clinical outcomes of patients with OLIF and TLIF. However, the restoration of disc height may affect the patient postoperative satisfaction.

\section{Declarations}

\section{Availability of data and materials}

We state that the data will not be shared since all the raw data are present in the figures included in the article.

\section{Ethics approval and consent to participate}

Non.

\section{Acknowledgements}

Not applicable.

\section{Author Contributions}


Conception and design, data acquisition, and drafting of the manuscript: Taibang Chen and Xiaoqing He; Analysis of the data: Taibang Chen; Critical revision and supervision: Taibang Chen and Xiaoqing He.

\section{Consent for publication}

Not applicable.

\section{Competing interests}

The authors declare that they have no competing interests.

\section{Funding}

No funding source was involved in the conduction of this study.

\section{References}

1. Ralph J Mobbs, Kevin Phan, Greg Malham, Kevin Seex, Prashanth J Rao. Lumbar interbody fusion: techniques, indications and comparison of interbody fusion options including PLIF, TLIF, MI-TLIF, OLIF/ATP, LLIF and ALIF. J Spine Surg. 2015;1(1):2-18.

2. Harms $\mathrm{J}$, Rolinger $\mathrm{H}$. A one-stager procedure in operative treatment of spondylolistheses: dorsal traction-reposition and anterior fusion (author's transl). Z Orthop Ihre Grenzgeb 1982;120:343-7.

3. Tao Lan, Shi-Yu Hu, Yuan-Tao Zhang et al. Comparison Between Posterior Lumbar Interbody Fusion and Transforaminal Lumbar Interbody Fusion for the Treatment of Lumbar Degenerative Diseases: A Systematic Review and Meta-Analysis. World Neurosurg. 2018; 112:86-93.

4. Mayer HM. A new microsurgical technique for minimally invasive anterior lumbar interbody fusion. Spine 22, 691-699, discussion 700 (1997).

5. Silvestre C, Mac-Thiong JM, Hilmi R,Roussouly P. Complications and morbidities of mini-open anterior retroperitoneal lumbar interbody fusion: oblique lumbar interbody fusion in 179 patients. Asian Spine J. 2012;6:89-97.

6. Seung Won Park, Myeong Jin Ko, Young Baeg Kim, Jean Charles Le Huec. Correction of marked sagittal deformity with circumferential minimally invasive surgery using oblique lateral interbody fusion in adult spinal deformity. J Orthop Surg Res. 2020;15(1):13.1545-1547.

7. Yoshinao Koike, Yoshihisa Kotani, Hidemasa Terao, Norimasa Iwasaki. Comparison of Outcomes of Oblique Lateral Interbody Fusion with Percutaneous Posterior Fixation in Lateral Position and Minimally Invasive Transforaminal Lumbar Interbody Fusion for Degenerative Spondylolisthesis. Asian Spine J. 2020;12: 10.31616.

8. Chen Yilei, Zhu Zhihai, Wang Yukang et al. Effects of oblique lateral interbody fusion and transforaminal lumbar interbody fusion for lordosis correction in degenerative lumbar diseases. Chin Med J (Engl). 2018; 3:98(25):1990-1995. [in Chinese] 
9. Hah Yong Mum, Myeong Jin Ko, Young Baeg Kim, Seung Won Park. Usefulness of Oblique Lateral Interbody Fusion at L5-S1 Level Compared to Transforaminal Lumbar Interbody Fusion. J Korean Neurosurg Soc. 2019; 15: 10.3340.

10. Guang-Xun Lin, Kutbuddin Akbary, Vit Kotheeranurak et al. Clinical and Radiologic Outcomes of Direct Versus Indirect Decompression with Lumbar Interbody Fusion: A Matched-Pair Comparison Analysis. World Neurosurg. 2018;119:e898-e909.

11. Shi-Qiang Qin, Yu-qing Sun, Wen-zhuo Ni, Comparison of the outcomes of OLIF and TLIF in the treatment of regressed spondylolisthesis with lumbar spinal stenosis [J]. Journal of Practical Orthopaedics. 2018,24(7):628-632. [in Chinese]

12. Sun-Ren Sheng, Yi-Bo Geng, Kai-Liagn Zhou et al. Minimally invasive surgery for degenerative spondylolisthesis: transforaminal or oblique lumbar interbody fusion. J Comp Eff Res. 2020 Jan;9(1):45-51.

13. Qiang Zhang, Guo-dong Shi, Hong-Wei Ding et al. Short-term effects of oblique/transforaminal lumbar interbody fusion for degenerative lumbar spondylolisthesis. J Spinal Surg, 2019;17(6):369393.

14. Wei-zhuang Guo, Xin-jian Yang, Yu-chen Zheng, et al. Oblique side approach and transforaminal lumbar interbody fusion Comparison of curative effect on treatment of degenerative diseases of lumbar spine. Clinical Medicine. 2019; 39(10): 65-66. [in Chinese]

15. Harms J, Rolinger H. A one-stage procedure in operative treatment of spondylolisthesis: Dorsal traction-reposition and anterior fusion. 1982;120:343-7.

16. Ralph J Mobbs, Kevin Phan, Greg Malham, et al. Lumbar interbody fusion: techniques, indications and comparison of interbody fusion options including PLIF, TLIF, MI-TLIF, OLIF/ATP, LLIF and ALIF. J Spine Surg. 2015;1(1):2-18.

17. Zeng ZY, Xu ZW, He DW, Zhao X, Ma WH,Ni WF, et al. Complications and prevention strategies of oblique lateral interbody fusion technique. Orthop Surg. 2018;10: 98-106.

18. Hui-Min Lin, Ren-jie Zhang, Cai-Liang She. Radiographic and Clinical Outcomes of Oblique Lateral Interbody Fusion Versus Minimally Invasive Transforaminal Lumbar Interbody Fusion for Degenerative Lumbar Disease. World Neurosurg. 2019;122: e627-e638.

19. Schwab F, Ungar B, Blondel B, Buchowski J, Coe J, Deinlein D, DeWald C, Mehdian H, Shaffrey C, Tribus C, Lafage V. Scoliosis Research Society-Schwab adult spinal deformity classification: a validation study. Spine (Phila Pa 1976). 2012;37:1077-82.

\section{Figures}




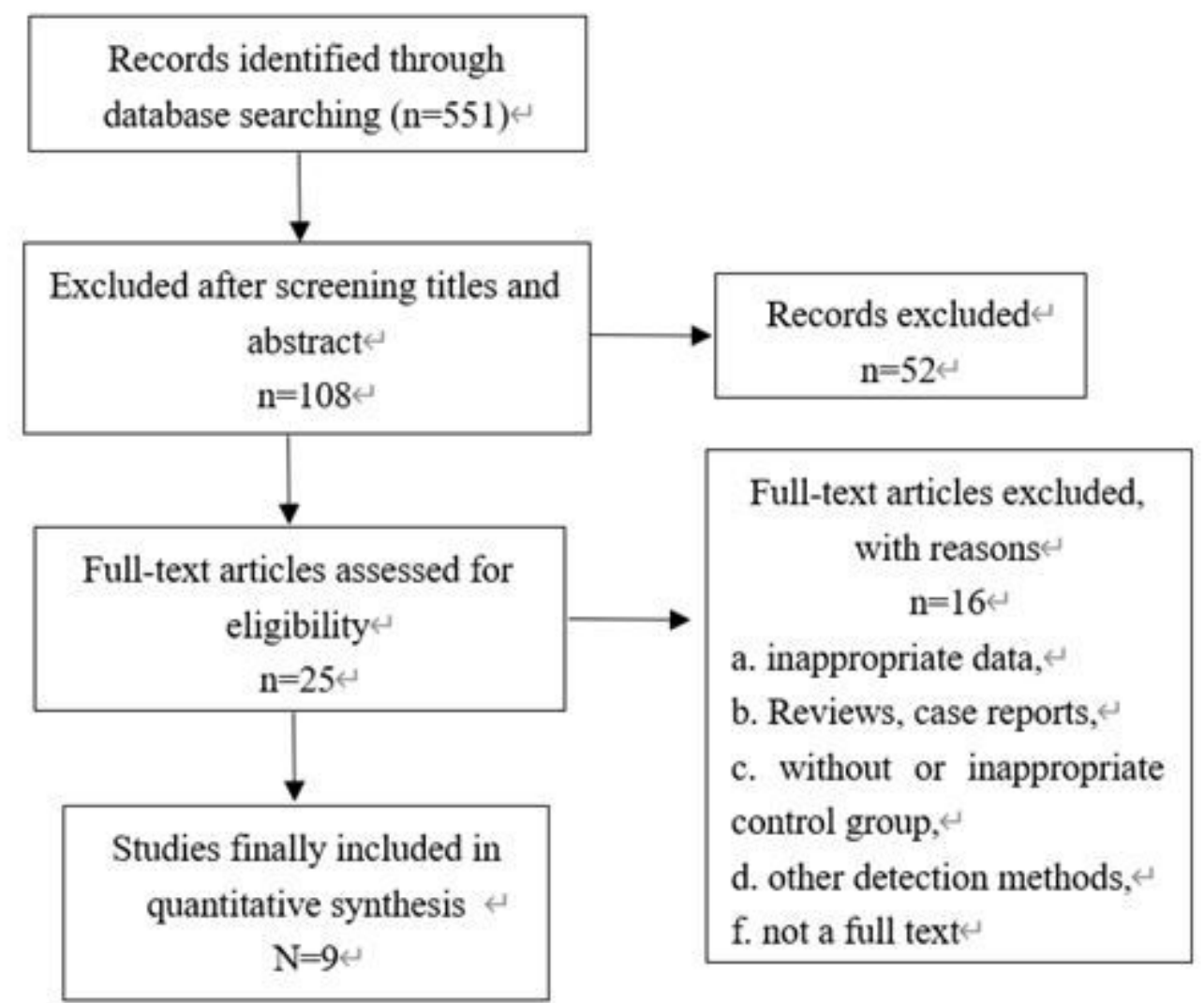

\section{Figure 1}

The search process and the reasons for exclusion

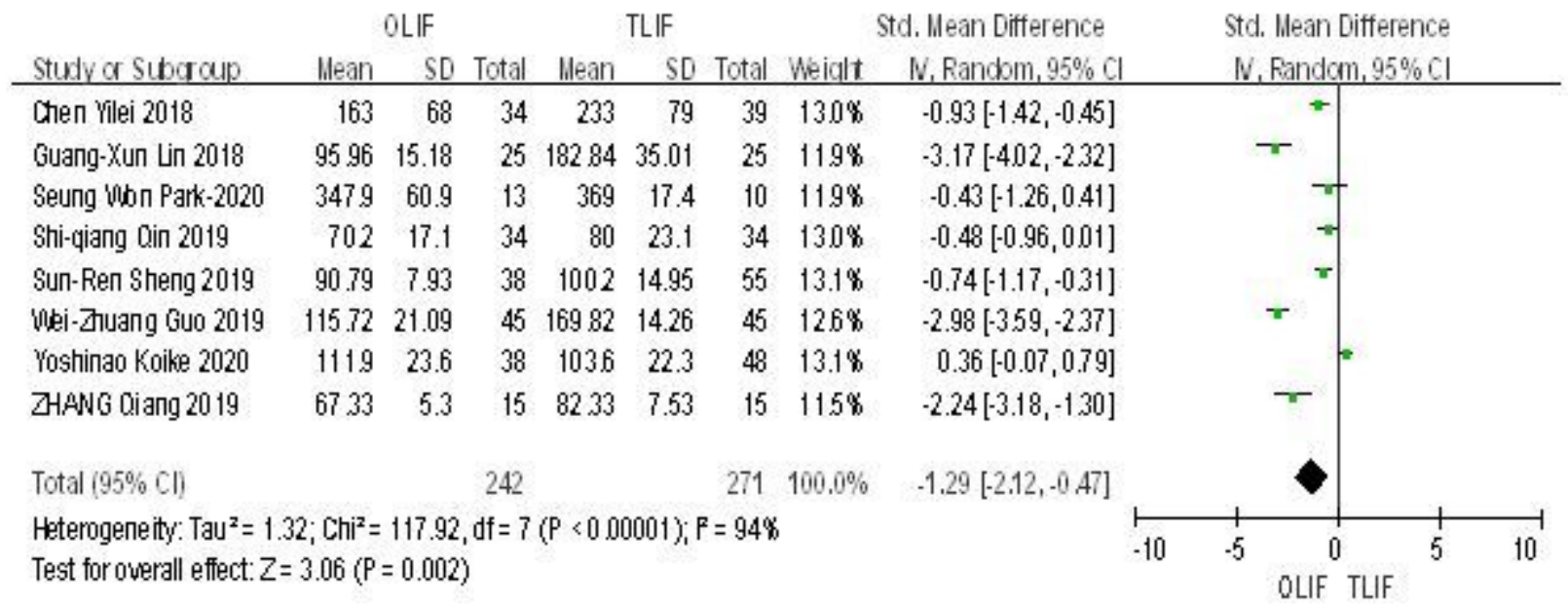

\section{Figure 2}

Forest plot for the operation time in the OLIF and TLIF groups 


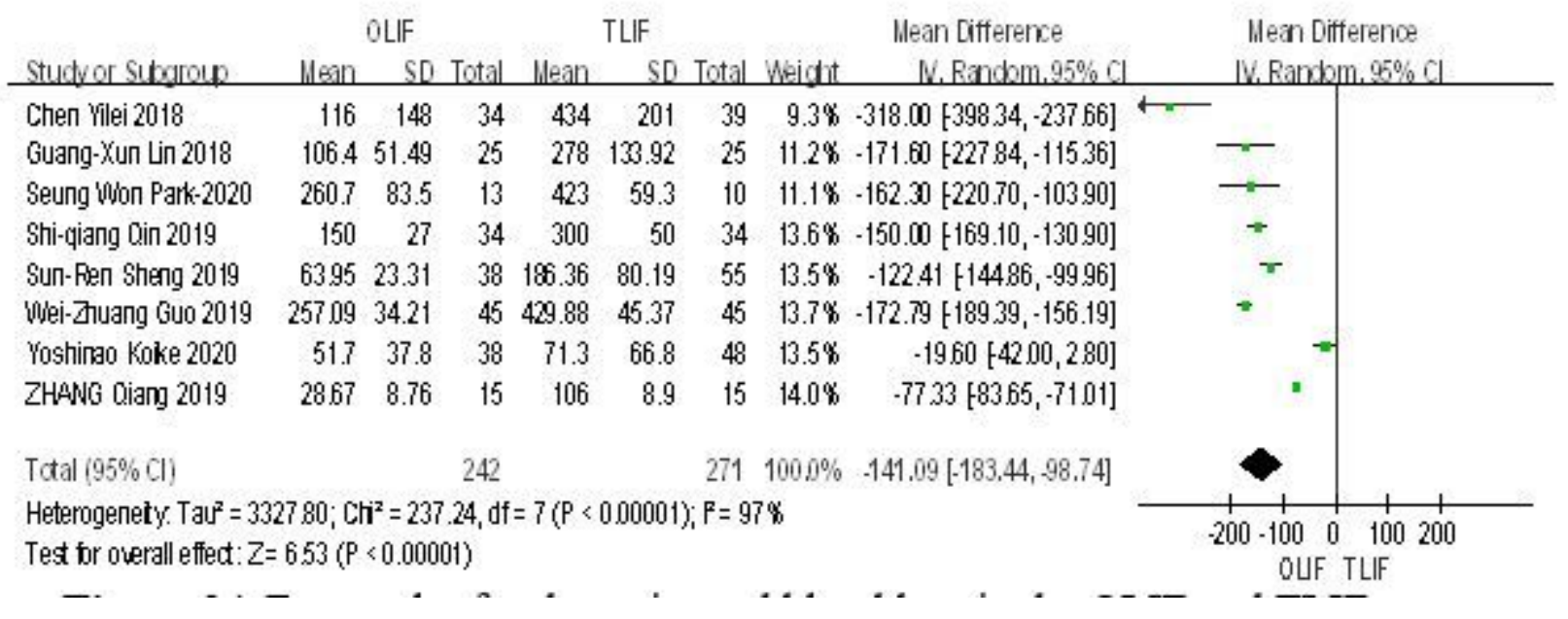

\section{Figure 3}

Forest plot for the estimated blood loss in the OLIF and TLIF groups

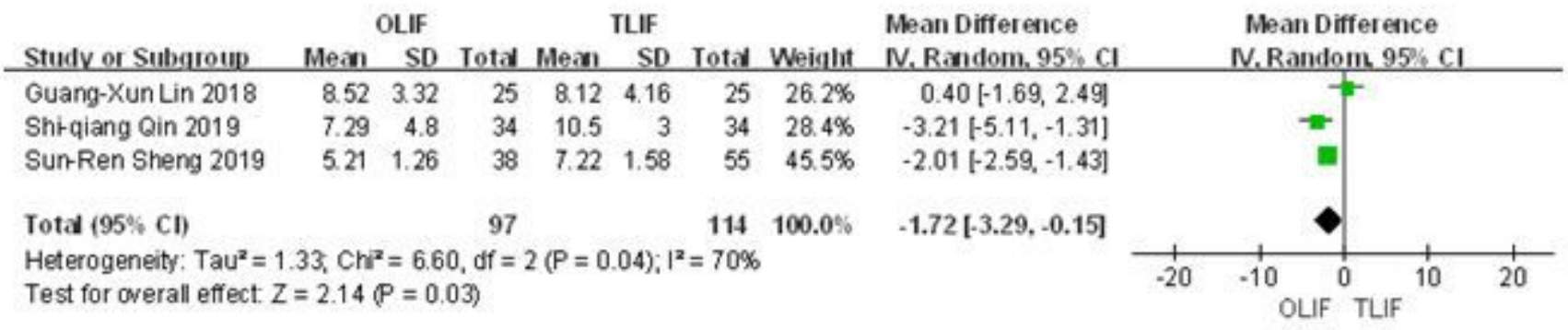

\section{Figure 4}

Forest plot for the length of hospital stay in the OLIF and TLIF groups

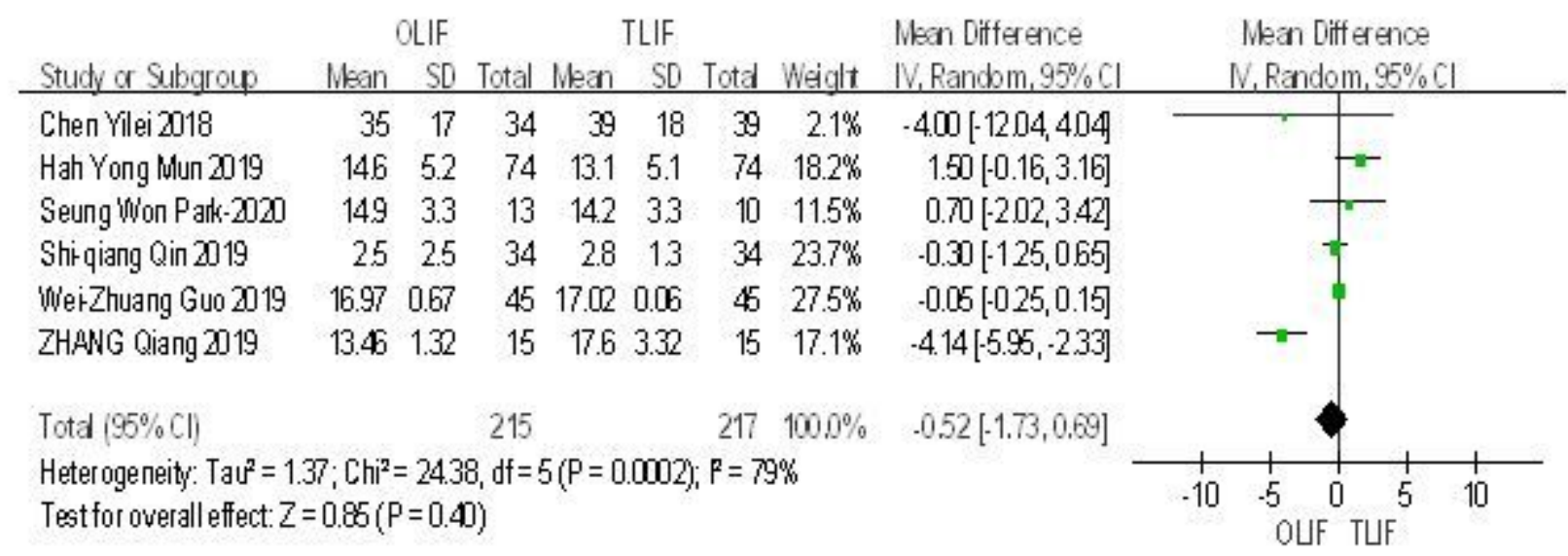

\section{Figure 5}

Forest plot for the ODI in the OLIF and TLIF groups 


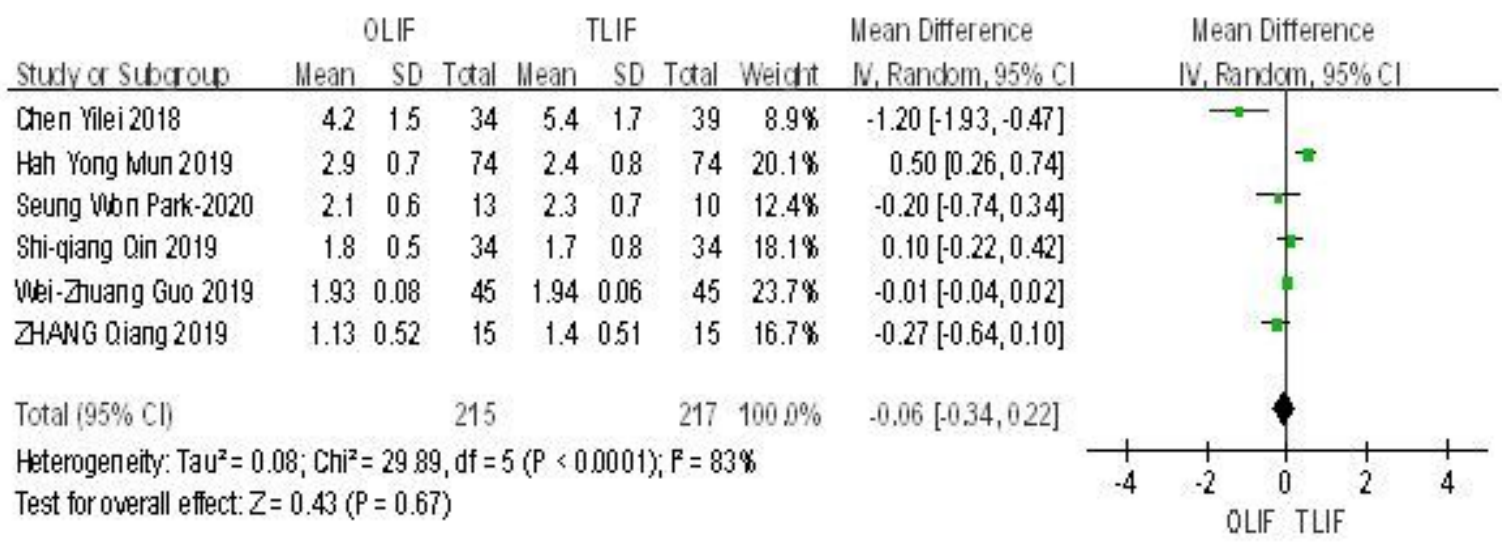

\section{Figure 6}

Forest plot for the VAS in the OLIF and TLIF groups

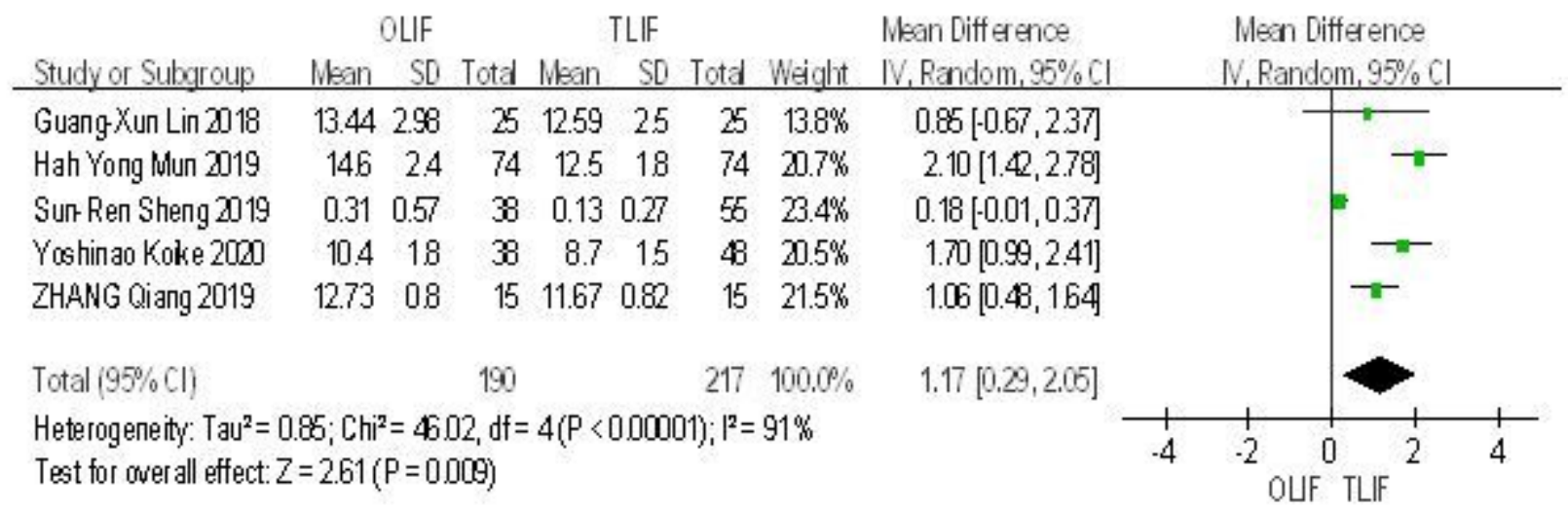

Figure 7

Forest plot for the disc height in the OLIF and TLIF groups

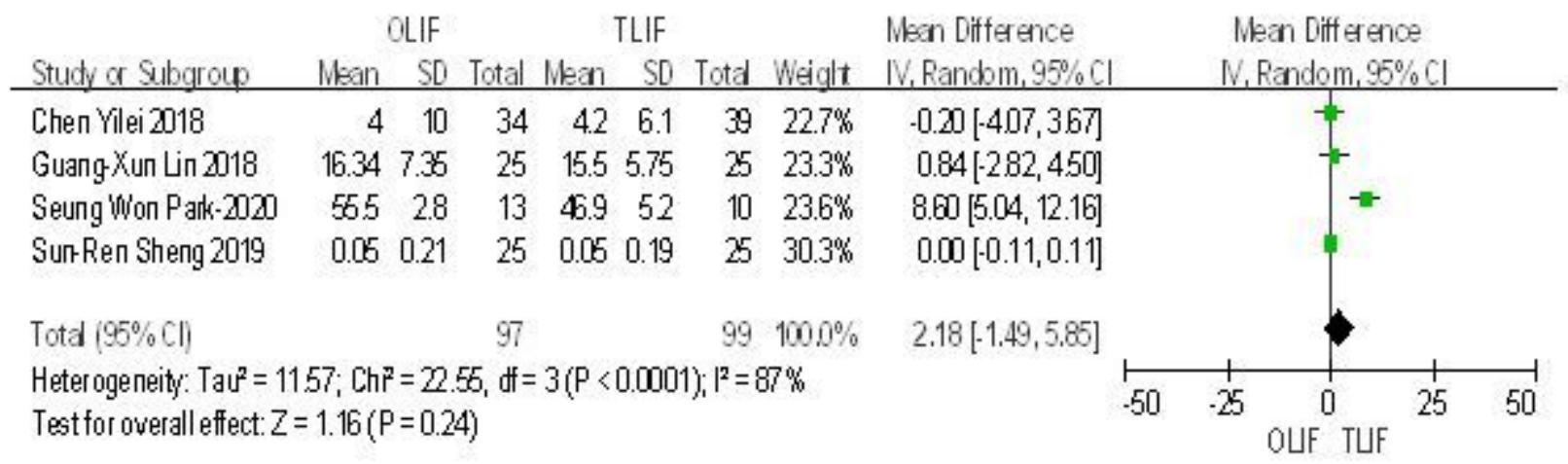

Figure 8

Forest plot for the disc height in the OLIF and TLIF groups 


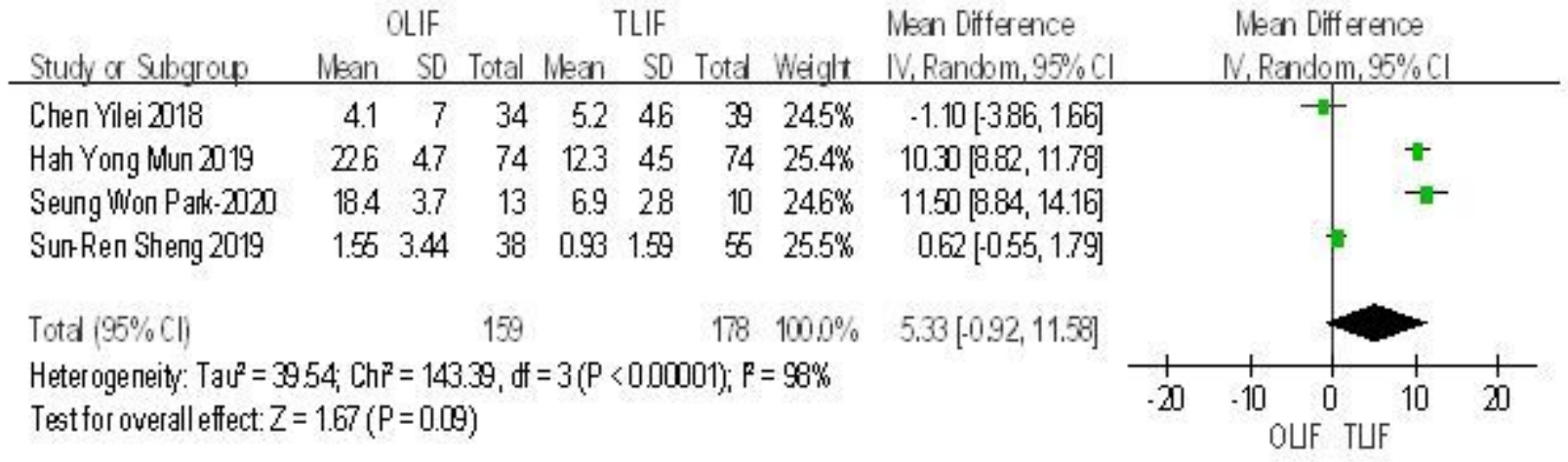

\section{Figure 9}

Forest plot for the disc angle in the OLIF and TLIF groups

\section{OUF TLF OddsRatio OddsRatio}

Study or Subcroup Events Total Everts Total Weiglt MH, Random $95 \% \mathrm{Cl}$ MH, Random $95 \% \mathrm{Cl}$

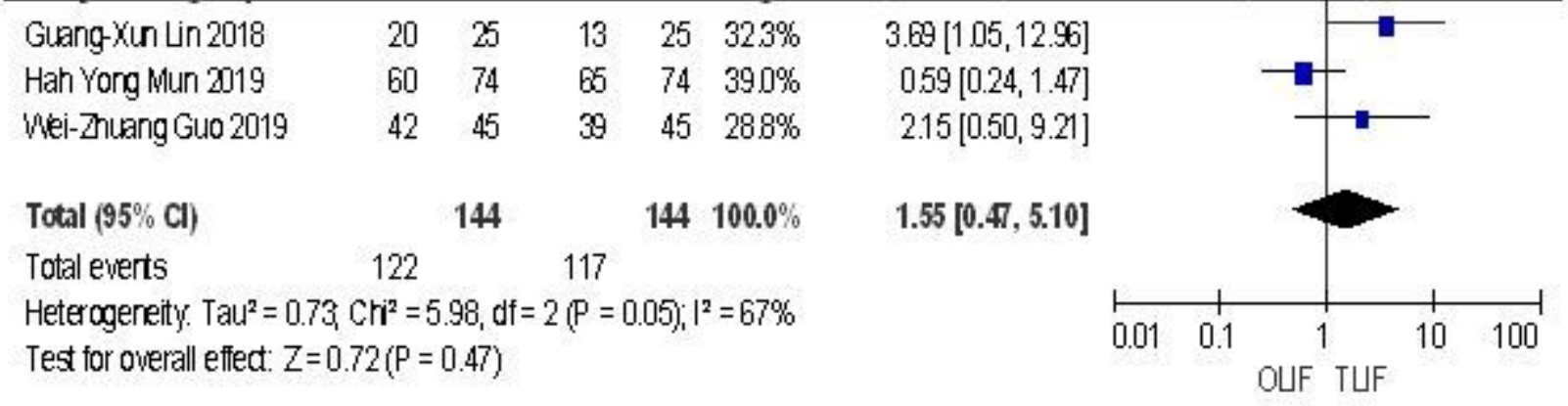

Figure 10

Forest plot for the fusion rate in the OLIF and TLIF groups

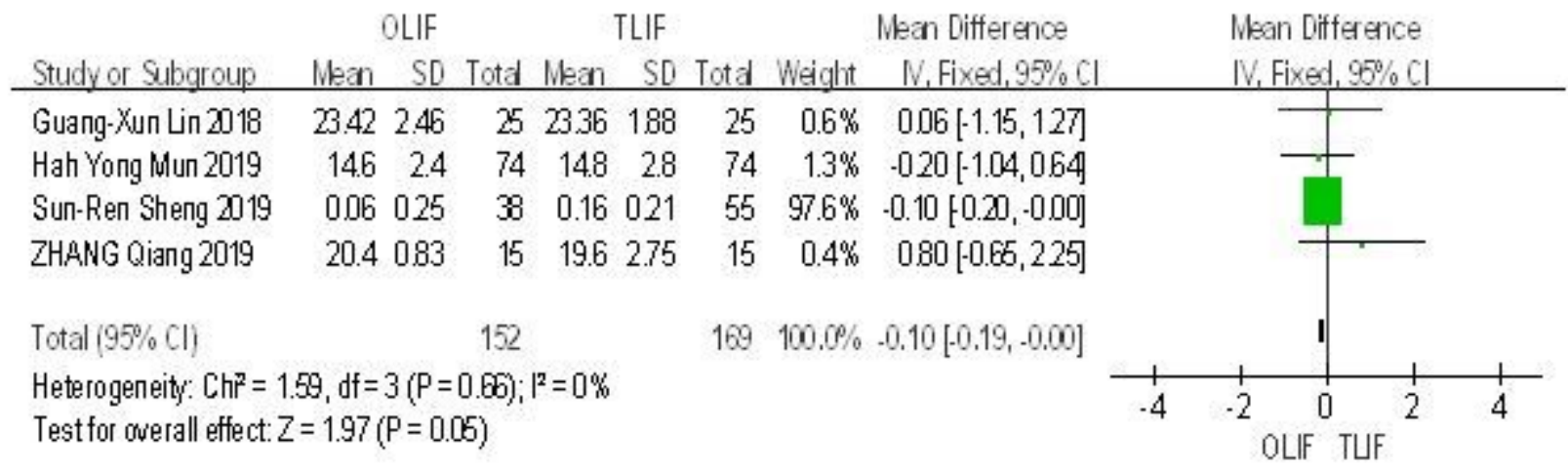

\section{Figure 11}

Forest plot for the foramina height in the OLIF and TLIF groups 